deprived and minority ethnic communities; maintain its vision to deliver and promote integrated services, and be flexible in possible developments.

\section{P6.067 FIVE YEAR SURVIVAL OF ADULT HIV-INFECTED PATIENTS INITIATED ON HAART AT THE AIDS SUPPORT ORGANISATION(TASO), UGANDA}

doi:10.1136/sextrans-2013-051184.1220

SSALI L, F Wasagaami, J Birungi, C Bakanda. The AIDS Support Organization, TASO, Kampala, Uganda

Background The AIDS Support Organization (TASO) Antiretroviral Program was rolled out in November 2004. The study aimed to assess adherence status of adult patients surviving for 60 months after HAART initiation in a resource poor setting.

Methodology Retrospective cohort study of adult patients who initiated HAART 60 months prior to the date of data abstraction at TASO Soroti, Eastern Uganda. Participants included in the study were male and female adult ( $\geq 14$ years) patients who initiated HAART at TASO Soroti (between $1^{\text {st }}$ August 2005 to $31^{\text {st }}$ March 2012). Since 2005 TASO Soroti has been providing HAART free of charge to patients either at World Health Organization (WHO) stage III or IV irrespective of CD4 cell count, or at any WHO stage with CD4 cell counts $\leq 350$ cells/ $\mu 1$.

Results Of the 4827 adults patients included in the analysis, $32.1 \%$ were male and the median age was 43 years. $67.9 \%$ were female and the median age was 41 years. $94.9 \%$ of patient were HAART naïve, $5.1 \%$ were transfer in. 3913(81.1\%) of 4827 patients were still on HAART after 5 years of follow-up. 18.9\% difference is attributed to lost to follow-up, patient transferred out and reported deaths. Among the patients with adherence assessment reported, $96.6 \%$ of patients had adherence level $>95 \%, 1.5 \%$ had adherence level of $85 \%-95 \%$ and $1 \%$ had adherence $<85 \% .74 .5 \%$ patients received their HAART refills at the CDDP, $23.8 \%$ received refills at the facility, and $1.4 \%$ received refills at their homes.

Conclusions These positive results after 5 years of initiating HAART in patients with advanced HIV disease demonstrate efficacy of HAART in resource-limited settings. Additional support is required to ensure timely HAART among adults.

\section{P6.068 EFFECT OF AN HIV PREVENTION PROGRAM AMONG FEMALE SEX WORKERS ON THE DECLINE IN SYPHILIS PREVALENCE AMONG THEIR CLIENTS IN SOUTH INDIA}

doi:10.1136/sextrans-2013-051184.1221

'RAJARAM S, ${ }^{2} \mathrm{M}$ Alary, ${ }^{3 P}$ Banandur, ${ }^{4} \mathrm{U}$ K Thammattoor, ${ }^{4} \mathrm{~T}$ Thomas, ${ }^{5} \mathrm{M}$ K Mainkar, ${ }^{5} \mathrm{R}$ Paranjape, ${ }^{6} \mathrm{R}$ Adhikary, ${ }^{2} \mathrm{~T}$ Duchesne, ' $\mathrm{S}$ Isac. ${ }^{1}$ Karnataka Health Promotion Trust, Bangalore, India; ${ }^{2}$ Centre de recherche, CHU de Québec, Québec, OC, Canada, ${ }^{3}$ National Institute of Mental Health and Neurosciences, Bangalore, India; ${ }^{4}$ St John's Research Institute, Bangalore, India; ${ }^{5} \mathrm{National}$ AIDS Research Institute, Pune, India; ${ }^{6} \mathrm{FHI}$ 360, Washington DC, WA, United States

Objective As a part of evaluating the Avahan Programme, two rounds of integrated biological and behavioural assessment (IBBA) surveys were conducted in 2006-07 and 2009-10 among clients of female sex workers (FSWs) across 17 districts in south India to measure the prevalence of HIV and STIs, and related risk factors ( $n \sim 7,000$ per round). We examined the association between changes in programme indicators and changes in syphilis prevalence.

Methods Using a random-effect multilevel logistic regression, we examined the effect of district-specific programme indicators for FSWs (from the Avahan computerised monitoring system) on the decline in syphilis prevalence among clients of FSWs after controlling for significant individual level variables. Program indicators included their 2006 value, the difference in their values between the surveys, and the interaction between the latter and study round. The model also controlled for baseline syphilis prevalence and its interaction with survey rounds.

Results Syphilis prevalence among clients of FSWs decreased from $4.8 \%$ (round 1 ) to $2.6 \%$ (round $2 ; \mathrm{p}<0.001$ ). The odds ratio (OR) of the interaction term between the difference in programme coverage (\% of FSWs covered by the programme in a given year) and the round was 0.983 ( $p=0.001)$, indicating that increase in coverage was significantly associated with the decrease in syphilis prevalence between rounds. ORs comparing syphilis prevalence between rounds varied with the level of increase in coverage and were statistically significant with coverage increase $\geq$ quartile $(\mathrm{Q}) 1: \mathrm{OR}=0.61$ at $\mathrm{Q} 1,0.53$ at $\mathrm{Q} 2,0.40$ at $\mathrm{Q} 3$ and 0.13 at $\mathrm{Q} 4$.

Conclusions The results support that the Avahan intervention among FSWs had a significant impact in reducing syphilis prevalence among their clients in settings covered by the intervention The triangulation of our results with those obtained among FSWs suggests a major impact of this intervention on the HIV/STI epidemic in southern India.

\section{P6.069 SOCIAL MOBILIZATION AND PEER-MENTORING TO ENCOURAGE VOLUNTARY COUNSELING AND TESTING AND POST-TESTING BEHAVIOR: A RANDOMIZED EXPERIMENT IN SENEGAL}

doi:10.1136/sextrans-2013-051184.1222

1J Graff Zivin, ${ }^{2}$ V Orozco, ${ }^{3} \mathrm{H}$ Thirumurthy, ${ }^{4} \mathrm{C}$ Sakho, ${ }^{5 \mathrm{P}}$ Diallo, ${ }^{2 \mathrm{M}}$ Offer Westort. ${ }^{1}$ University of California - San Diego, San Diego, CA, United States; 'World Bank, Washington, DC, United States; ${ }^{3}$ University of North Carolina - Chapel Hill, Chapel Hill, NC, United States; ${ }^{4}$ Ministry of Health, Dakar, Senegal; ${ }^{5}$ National AIDS Commission, Dakar, Senegal

Background To evaluate the effectiveness of two HIV/AIDS sensitization campaigns of the community-based organisations (CBOs) it financed, the National AIDS Commission of Senegal randomly assigned its financial support across the country's 14 regions, establishing three groups of health districts. Health districts where NACfunded $\mathrm{CBO}$ s carried out i) mass social mobilisation communication campaigns; ii) campaigns using peer-mentoring; and iii) control health districts, where CBOs didn't receive NAC support.

Methods We exploit the initial random assignment and differences at the time of implementation for the intervention groups (the randomization plan was not followed in the control group so we remove it from the analysis). We use two methods. First, a beforeafter analysis where we examine trends to estimate the change in outcomes during each of the four quarters following implementation of the intervention, relative to the average of the four quarters before the intervention. Second, we estimate a difference-in-differences regression model to directly compare the effectiveness of each intervention.

Results The before-after analysis shows that immediately after the intervention was completed, peer-mentoring doubled the number of adults who were tested for HIV and the number of adults who picked up their test results. For both outcomes, the number tripled three quarters later. Social mobilisation was less effective over time. For those individuals who test HIV-positive, peer-mentoring induces more HIV-infected individuals to collect their results and engage in post-test counselling. However, the second method finds no significant differences between the two intervention groups.

Conclusion These results suggest that both interventions are successful for the general population and to a lesser for the HIV-infected population. The difference-in-differences analysis suggests that there is little reason to favour one intervention over the other. Analysis at the health district level greatly diminishes our power to detect changes, especially for social mobilisation activities. 